\title{
Regeneration and Recovery in the Fetal Nervous System after Radiation Injury
}

\author{
Constance J. D'Amato ${ }^{1}$ \\ Department of Pathology, Box 45, The University of Michigan Medical Center, \\ Ann Arbor, Michigan 48109
}

Received February 27, 1981; revision received December 28, 1981

\begin{abstract}
Previous studies showed that recovery from destructive injury in 12-day fetal rats ( 23 to 34 somite pairs) exposed to $150 \mathrm{R}$ appeared to be remarkably good except for minor abnormalities of the brain and spinal cord in some animals and occasionally microphthalmia. Nothing more was known of the morphogenesis of this restitution. A series of rats irradiated on the 12 th fetal day was studied histologically and in other ways from a few hours after exposure to adulthood. Five to eight hours after $150 \mathrm{R}$ there was widespread necrosis of certain classes of primitive proliferative cells in the nervous system and other tissues, but residual cells had resumed proliferative activity. By $24 \mathrm{~h}$ regeneration of the proliferative cells was advanced and most necrotic cells had disappeared, and at $48 \mathrm{~h}$ the irradiated fetus closely resembled the normal. There were very few residual dead cells, and minute foci of proliferative cell rosettes were present in the midline of the ventral spinal cord and brain stem. Seventy-two and ninety-six hours after irradiation the fetuses looked like their normal counterparts except for persistent or resolving minute rosettes. At later stages to adulthood, effects of the rosette formation were not visible. In some individuals the spinal cords were reduced slightly in overall cross-sectional area and the pallium was shorter than normal. Other irradiated animals could not be distinguished from normals.
\end{abstract}

\section{INTRODUCTION}

Prenatal irradiation of rats with single exposures of 150 or $200 \mathrm{R}$ in the period of first somites to the early postnatal period usually results in malformations $(1-7,11-14)$. The highly reproducible abnormalities are the results of a balance between the effects of cell-killing and other injury and the regulative regenerative capacity of surviving cells to restitute the injury. Early observations $(5,14)$ showed that albino rats exposed to $150 \mathrm{R}$ during

${ }^{1}$ This work is supported by U.S. Public Health Service grant NS10531. 
the 12 th fetal day, at about 28 to 34 somite pairs, resulted in animals that appeared superficially normal. The isocortex of their brains, however, ranged from about three-quarters to seven-eighths normal thickness, and the posterior medial vertex of the pallium was slightly deficient. Recent studies with another strain of rats exposed to $150 \mathrm{R}$ during the 12 th fetal day, at maturation showed less abnormality, their brains approaching the normal in gross and microscopic appearance (12). Horseradish-peroxidase histochemistry showed abnormalities of some corticospinal neurons, and a few animals showed eye, spinal cord, and other abnormalities. Locomotor functions appeared normal (1). How this degree of recovery from severe fetal radiation injury came about has not been studied. This paper describes the recovery and examines the adult stages further. A preliminary report was made (2).

\section{METHODS}

Animals. "Irish" rats (black with white feet), the F1 cross between albino females of Wistar origin, brother-sister mated for about 85 generations, and black-selfed nonagouti males, brother-sister mated for about 75 generations, were the principal animals (1). Care and housing of the animals were fully accredited by the American Association for Accreditation of Laboratory Animal Care. The experiments conformed to standards in the Guide for the Care and Use of Laboratory Animals, U.S. Department of Health, Education and Welfare Publication 73-23, 1972.

Radiation Factors and Staging of Fetuses. These have been published in detail (1).

Anatomic and Histologic Methods. A reference collection of normal developing Irish rats, consisting of serially sectioned whole fetuses for each day (9 to 19) and the brains and levels of spinal cords of older fetuses and infants to a week after birth, juveniles, and mature animals, was used for comparisons with irradiated animals.

The litters of six pregnant rats exposed to $150 \mathrm{R}$ on the $12 \mathrm{th}$ fetal day, totaling 38 animals, were studied histologically. The fetuses were removed surgically ("Caesarean section") as described earlier $(1,14)$. Stages examined were (i) 5, 6, and $8 \mathrm{~h}$ after irradiation, (ii) days 13 to 17 and day 21 of gestation, (iii) 7 and 10 days old, and (iv) 1, 2, 4, 9, and 18 months of age. Two or more fetuses sampled at each stage were fixed in Bouin's solution, paraffin embedded, serially sectioned at $8 \mu \mathrm{m}$ in sagittal or frontal planes, and stained with hematoxylin and eosin. Three adult brains were similarly prepared, and every tenth section, or every section in regions of special interest, was stained with cresyl violet or hematoxylin and luxol fast blue. The brain of one adult animal was stained by a modified Golgi 
method (9). The corticospinal neurons in five 7- or 10-day-old, one 1month-old, and three adult animals were studied by the retrograde horseradish-peroxidase method in serial sections $(1,10)$. Normal controls were prepared for all of these irradiated animals.

Comparisons of Irradiated with Normal Animals. Comparisons of living animals and their nervous systems and other organs seen at autopsy was aided by photography. Special attention was given to fixation, dehydration, and microtome compression in the histologic comparisons. Although it is impossible to align in exactly the same way two adult brains or fetuses for sectioning, good comparisons could be made by matching various parts of the subjects throughout the serial sections $(1,10,12)$.

\section{RESULTS}

The sequence of changes following irradiation are described beginning on the 12th fetal day a few hours after $150 \mathrm{R}$, then 1 to 5 , and 9 days later, and in the infant, juvenile, and adult stages. The forebrain, especially the dorsal pallium, is used to illustrate the progress of these alterations (Figs. 1-6).

Twelve Days: 5,6 , or 8 Hours after Irradiation. The normal 12-day fetus of 28 to 34 somite pairs has a forebrain which is just beginning to divide into hemispheres, and the lungs and liver are just beginning to form (Fig. 1A). In ten 12-day fetuses examined 5,6 , or $8 \mathrm{~h}$ after $150 \mathrm{R}$, there was extensive necrosis of primitive cells. In most parts of the central nervous system an estimated one-half of the primitive cells, and in some places as many as three-quarters of them, were killed. The disintegrated nuclei of these necrotic cells were principally in the outer two-thirds of the cerebral vesicle, but in places some were also close to the ventricular surface, especially where the continuity of the ventricular lining was briefly disrupted. Some dead cells escaped into the ventricle (Fig. 1B). Mitoses, known to have ceased during the first 3 or $4 \mathrm{~h}$ after exposure $(7,11,13,14)$, had resumed.

The severest destruction in the nervous system occurred in the dorsal pallium. Necrotic cells were seen in nearly as great numbers in the lateral parts of the pallium, the optic pit regions of the diencephalon, the lateral walls of the diencephalon, the middorsal part of the midbrain, the spinal cord, and the proximal parts of the developing spinal root ganglia. Similar destruction occurred in populations of primitive mesenchymal cells, especially perinephric, facial, and limb-bud regions, and in the dermatome parts of the somites. Occasional phagocytes containing dead cells were seen in the 8-h exposed fetuses in regions of greatest mesenchymal necrosis, and in the distal parts of the spinal ganglia at their borders with the mesenchyme. In contrast, there was sparing of much of the base of the dien- 

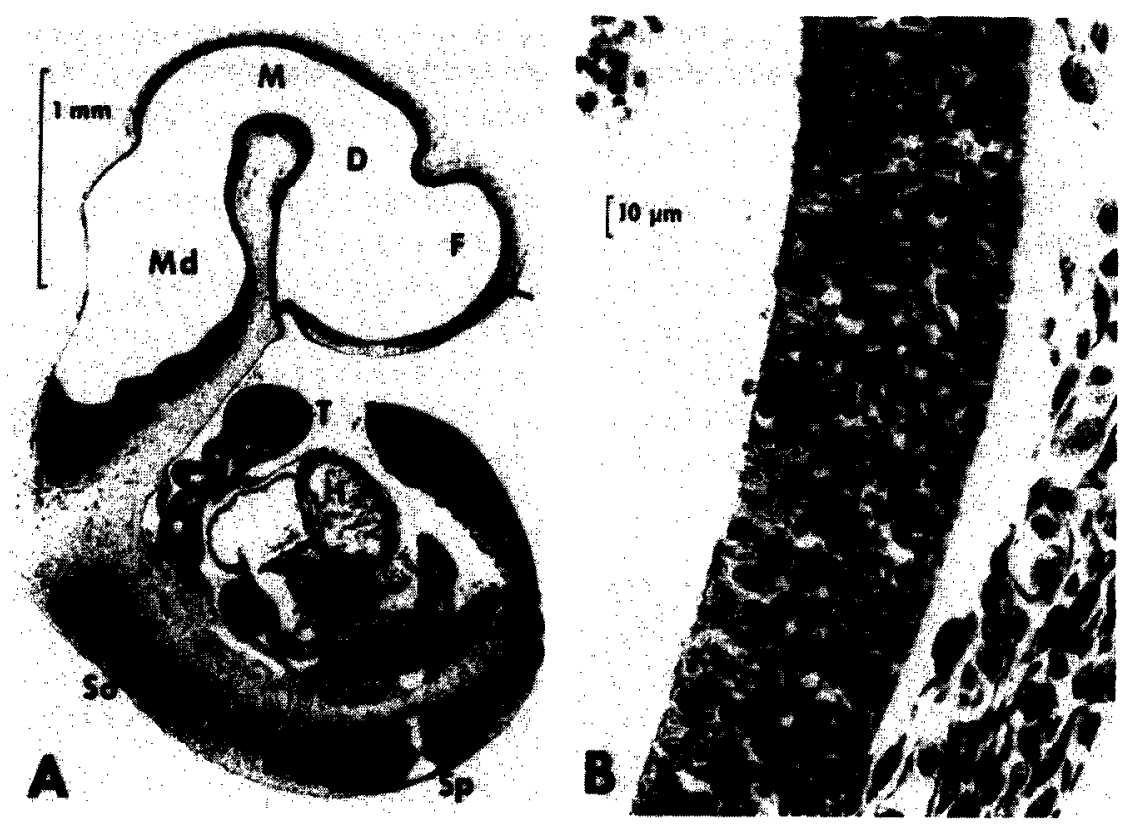

FIG. 1. Response of primitive cells in the pallium of a 12-day fetus $6 \mathrm{~h}$ after $150 \mathrm{R}$. Alow magnification, sagittal view, of the fetus for orientation. Arrow indicates the dorsal pallium of the forebrain. F, shown in high magnification in B. D-diencephalon, M-midbrain, Mdmedulla, So-somites, Sp-spinal cord, $\mathrm{T}$-tongue, and $\mathrm{H}$-heart. Hematoxylin and eosin. B-acute necrosis of primitive cells in a segment of the pallium, which appear as numerous coarse black dots. A few have escaped into the ventricle, upper left. Mitoses have resumed in cells lining the ventricle.

cephalon, medulla, midbrain, the more mature posterior root ganglion cells, the heart, and the epithelium of the integument and enteric system. Initial stages of rosette formation $(11,14)$ in the periventricular proliferative cell populations were seen only in the cervical and lumber spinal cord and at the site of the future interpeduncular space.

Thirteen Days: 1 Day (24 Hours) after Irradiation. In four fetuses the majority of dead cells had disappeared everywhere except in the spinal cord and parts of the medulla. Those that remained were being displaced toward the periphery by the regenerating proliferative cells. The nuclei of the proliferative cells in the irradiated fetuses were larger with less densely concentrated chromatin than in normals (Fig. 2). Remnants of residual necrotic cells were seen in the few phagocytes that had developed in various places (Fig. 2A). The fetuses had grown in the 24-h period despite the radiation injury, but lagged behind the normal 13-day fetuses (B). Minute rosettes of the proliferative cells had developed in the sites where they were being initiated on the 12 th day. 

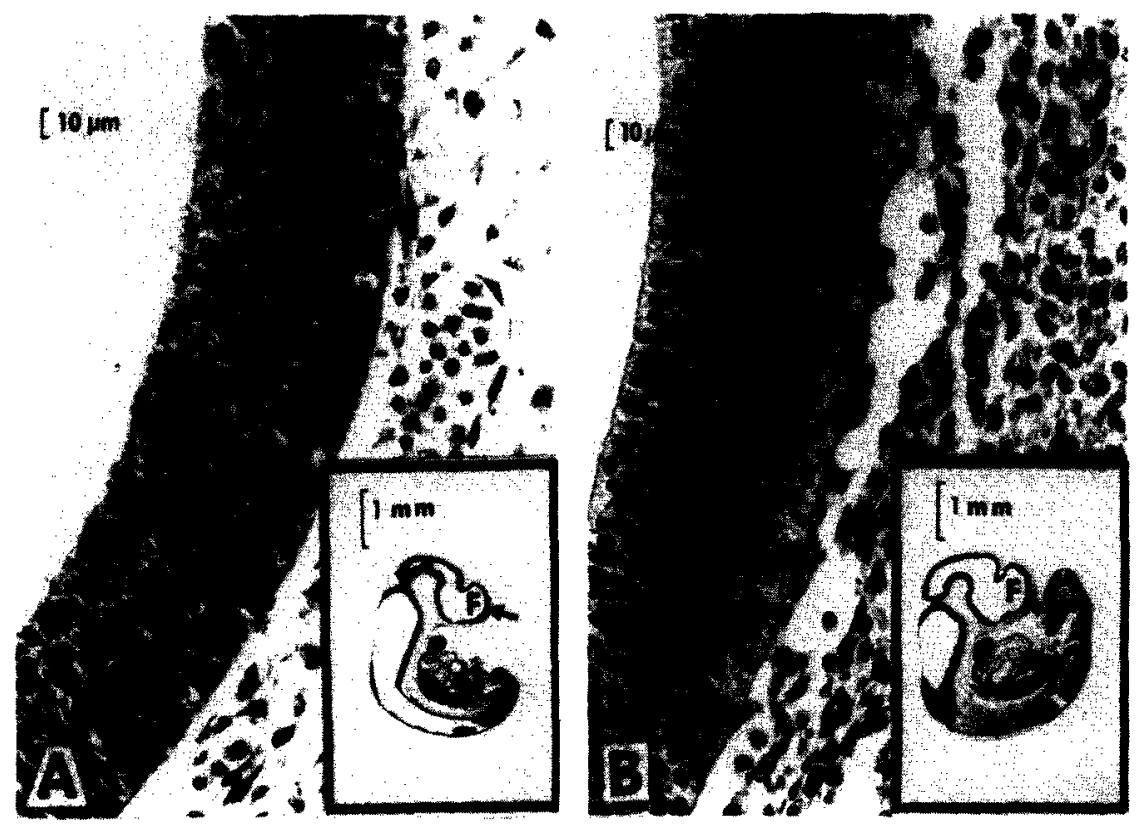

Fig. 2. A-the dorsal pallium of a 13-day fetus $24 \mathrm{~h}$ after $150 \mathrm{R}$. B-a normal fetus. Hematoxylin and eosin. The inserts show the respective fetuses in sections oriented sagittally through their forebrains. Insert arrows indicate the dorsal pallium of the forebrain, F, a segment of which is shown at high magnification. There has been substantial growth and recovery. A few residual necrotic cells persist (black dots), some clustered in a phagocyte in the upper part of the figure (arrow). Cell nuclei of the irradiated pallium are larger than those of the normal pallium.

Fourteen Days: 2 Days (48 Hours) after Irradiation. In two fetuses there were almost no residual dead cells (Fig. 3A); a few dead cells persisted in phagocytes in the periphery of the spinal cord. As a whole, the fetuses resembled their normal 14-day counterparts, but as Fig. 3 A and B show, the cells of the pallium appeared to be less orderly in their arrangement than normal. In one fetus there was a minute dorsal outpocketing of the third ventricle, and in both there were minute rosettes corresponding to those seen in earlier stages.

Fifteen Days: 3 Days $(72$ Hours) after Irradiation. The one fetus at this stage showed no residual dead cells or phagocytes and could not be distinguished in serial sections from normal fetuses.

Sixteen Days: 4 Days (96 Hours) after Irradiation. Four animals were generally similar to their normal counterparts, except for a number of focal abnormalities in the nervous system. At the sites of rosette formation in the spinal cord seen in earlier stages, there were now small foci of irreg- 

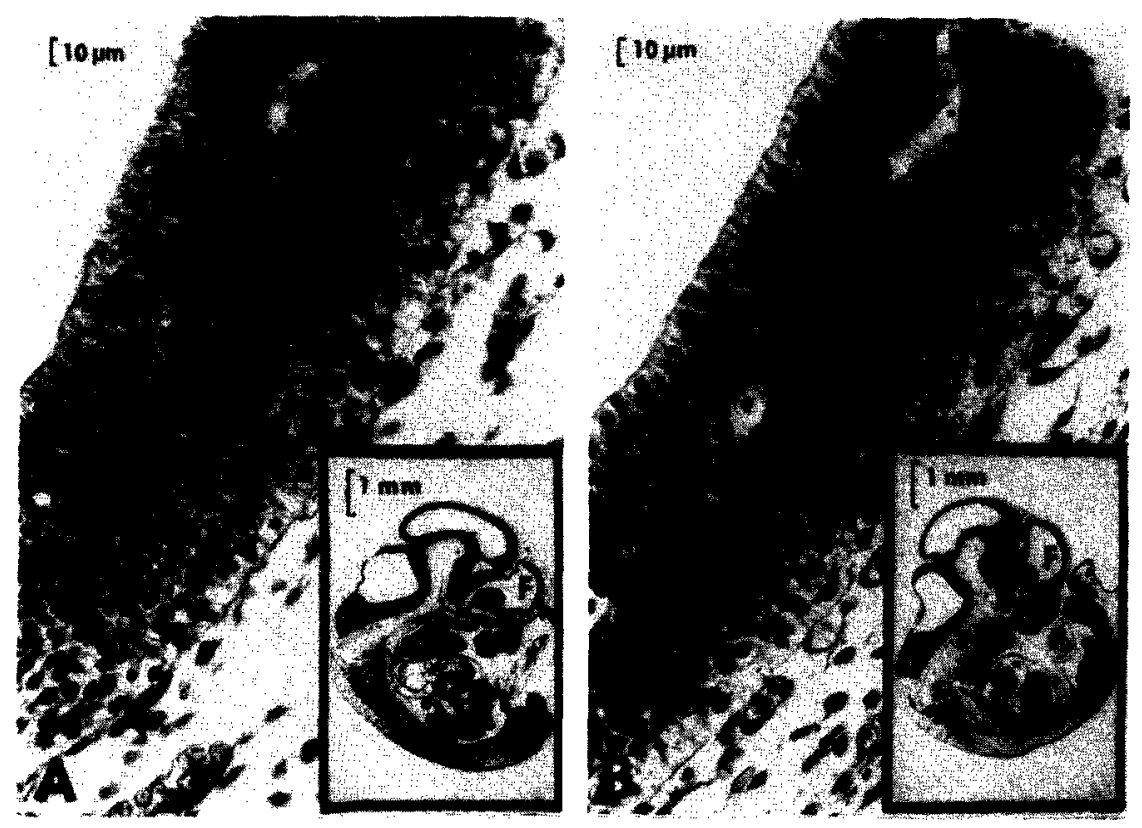

Fig. 3. A-the dorsal pallium of a 14-day fetus $48 \mathrm{~h}$ after $150 \mathrm{R}$. B-a normal pallium. Hematoxylin and eosin. The inserts show the respective fetuses in sections oriented sagittally through their forebrains. Arrows indicate the dorsal pallium of the forebrain, $F$, a segment of which is shown at high magnification. The cells in the irradiated pallium appear somewhat less ordered than those in the normal. Necrotic cells have largely disappeared.

ularly arranged neurons and ependymal cells. In the dorsal pallium of the irradiated fetus, the proliferative and differentiating cells of the early cortex were indistinguishable from normal (Fig. 4). The forebrain as a whole appeared very slightly shorter in the irradiated animal as shown in the insets; the apparently thicker occipital pallium in the normal is a result of differences in alignment of the two fetuses.

Seventeen Days: 5 Days after Irradiation. In two fetuses there were resolved rosettes in the spinal cord like those seen in the 16-day fetuses. No other abnormalities could be detected; the forebrains could not be distinguished from normal.

Twenty-one Days: 9 Days after Irradiation. The dorsal pallium of the two irradiated rats was not quite as mature as that of the normal animals, and might have reflected a small discrepancy in ages, but the irradiated pallia were about 20 and $10 \%$ shorter than those of the controls. The diencephalons, however, could be almost exactly superimposed. The junctions of the diencephalons with the midbrains were a little shorter than that of the normal animals. 

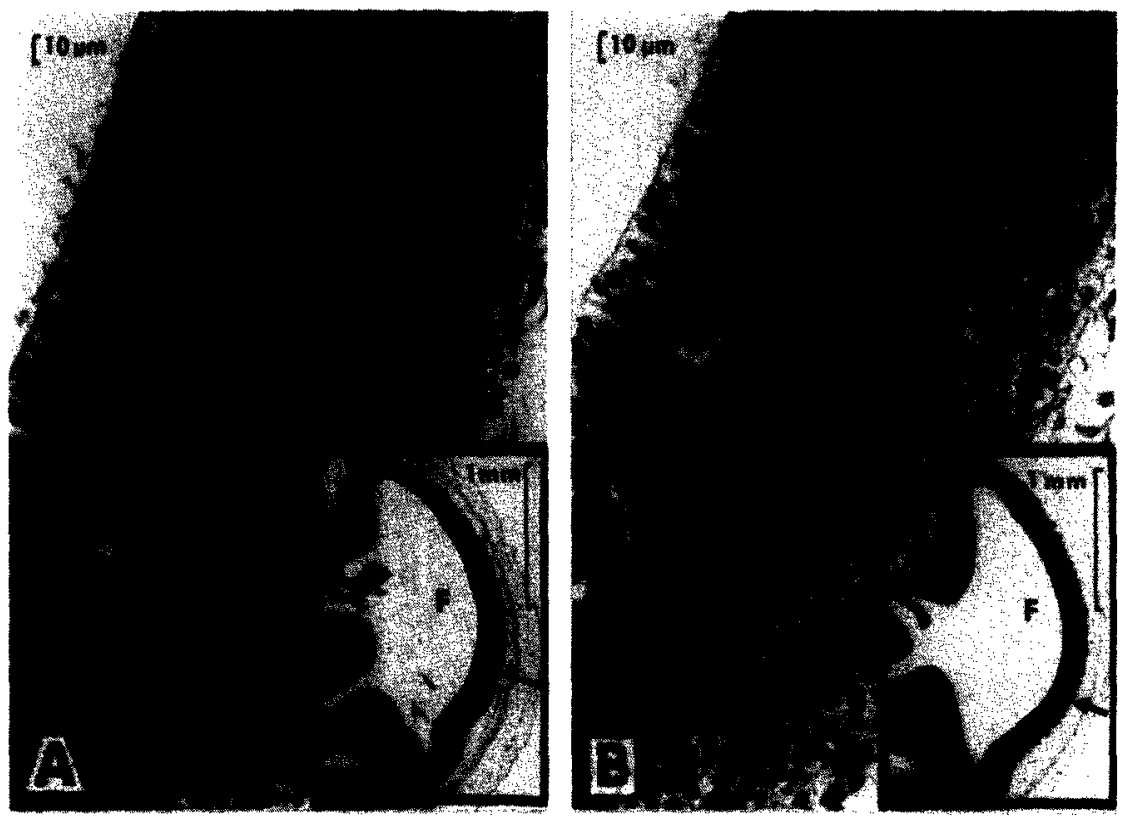

FIG. 4. A-the dorsal pallium of the 16-day fetus $96 \mathrm{~h}$ after $150 \mathrm{R}$. B-a normal pallium. Hematoxylin and eosin. The inserts show the respective forebrains, $F$, of the fetuses. The arrows indicate the segments of the dorsal pallium shown at high magnification. There was no difference between the fetuses. The thicker posterior pallium of the normal rat (upper part of the insert) was attributed to the angle of section.

Comparisons of the spinal cords in cross sections showed that there was a small reduction in the areas of the ventral horns, including the domains of the anterolateral and posterolateral cell groups. Products of early rosette formation were not recognized. All of these findings corresponded to those observed in some mature rats described previously $(1,12)$.

Infant, Juvenile, and Adult Stages after Irradiation on the 12th Fetal Day. Eighteen rats were observed in various ways at these stages. Behavioral studies (motor function) in adults, histochemical demonstration of the postnatal development and adult appearance of corticospinal neurons, and preliminary observations on gross anatomy and histologic appearance of the nervous system were reported previously (1). Further study showed that in three of the seven mature brains examined, the pallium was only slightly shorter than normal in greatest longitudinal dimension, but the medial parts of the occipital pallium were deficient enough to expose the inferior colliculi as shown in Fig. 5A. The olfactory bulbs were a little shorter in the irradiated rats, but they were of normal width. In contrast 

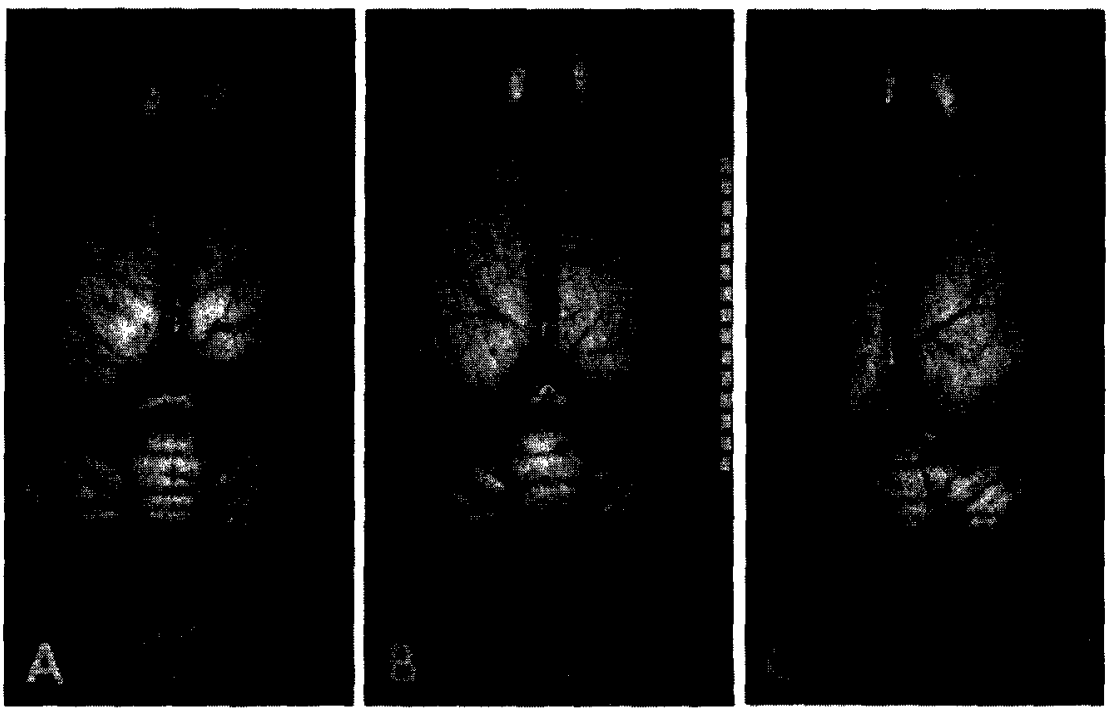

FIG. 5. The fixed brains of two adult rats, $A$ and $B$, that were exposed to $150 \mathrm{R}$ on the 12 th fetal day are shown in dorsal view compared with a normal, $C$. The inferior colliculi were uncovered in A, and slightly visible in B. The olfactory bulbs in A and B were slightly shorter than normal. The millimeter rule was photographed with the brains.

to the brains in Fig. 5, the brain of the irradiated rat in Fig. 6 was not distinguishable from normal, either grossly or histologically.

\section{DISCUSSION}

Regeneration and Restitution. The remarkable capacity for recovery after destructive injury, and the rapidity with which it was accomplished in the rat fetuses, is usually considered to be restricted to simpler vertebrate embryos, such as amphibia and birds. An earlier study in this laboratory (4) showed, however, that rats in early neural fold and somite stages could almost wholly restitute the devastating cell loss caused by $150 \mathrm{R}$, paralleling the restoration demonstrated in amphibian embryos when one of their neural folds was extirpated experimentally (15). The 12-day rat fetus is in a substantially more advanced stage than those of the simpler vertebrates that have been used to illustrate the regulative capacity of primitive cells. It is reasonable to assume, however, that although the prospective fate of many of its neural and nonneural proliferative cell populations had been determined by this time, these populations retained characteristics of morphogenetic fields, that is, they still possessed regulative properties. A morphogenetic field has been defined as the smallest unit of tissue that can 

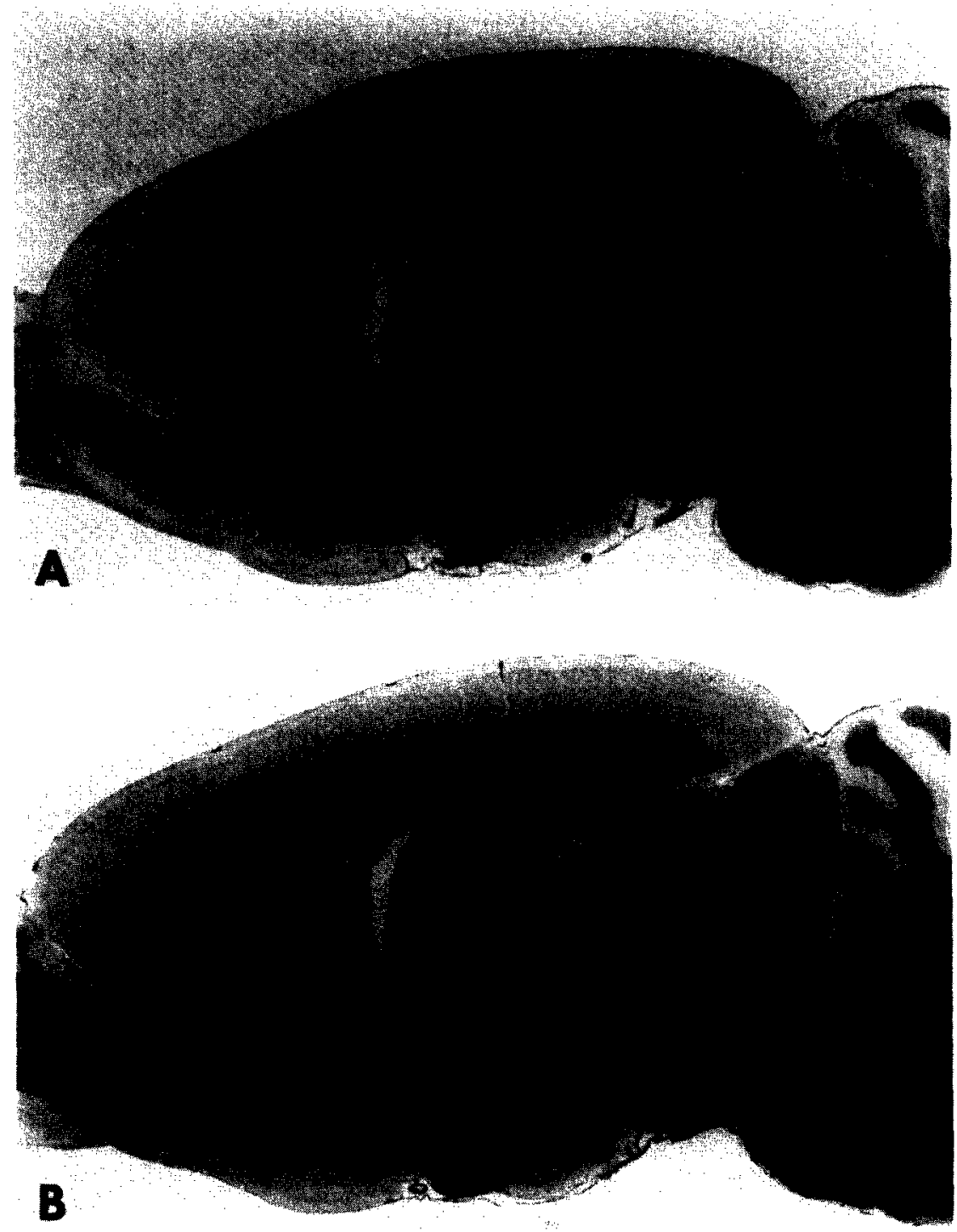

FIG. 6. A-the brain of an adult rat exposed to $150 \mathrm{R}$ on the 12 th fetal day is shown in sagittal histologic section. B-a normal brain. Luxol fast blue and cresyl violet. The sections were closely matched except that the frontal part of the irradiated brain was in a more lateral plane. Grossly and in histologic sections the brains were not distinguishable.

form an entire structure in situ or in isolation (15). If the whole population is destroyed the structure cannot form, but if some cells of the population remain, their regulative properties permit them to form the structure. Why 
they could do this so nearly perfectly after injury on the 12 th fetal day, but not at other stages such as the 11 th or 13th day, when deficits and malformation result $(3,11)$, is not known.

Assessing Normality and Abnormality. How closely do some of the 12day irradiated rats approach the normal rat? The answer lies in the limitations in defining normal and abnormal. Aligning whole fetuses and brains in precisely the same framework for serial sections time after time is impossible, but comparison of multiple sections of the normal and abnormal subjects allows a reasonably good approximation. The characteristics of a normal specimen are judged to be normal because they represent a range that experience has accepted. Undoubtedly significant differences between normal and abnormal animals go unrecognized in a study of this kind. A deficiency of the medial occipital parts of the cerebral hemispheres in some 12-day irradiated rats caught the eye because even slightly uncovered colliculi are noticeable. An equal deficiency of some other part of the hemispheres without well defined reference could easily go unnoticed. At autopsy the various organs of these animals appeared to be normal except for the brain, spinal cord, and eyes, as noted.

Selective Cell-Killing. The primitive cells killed rapidly by 150 or 200 $\mathrm{R}$ in the rat from about the ninth fetal day to the end of the first postnatal week have been shown to be principally in Gl (postmitotic primitive proliferative and differentiating) and $\mathrm{G} 2$ (prophase) phases $(7,8,13)$. The $\mathrm{S}$ (synthesis) phase cells are largely spared in this dose range. By irradiating a rat at a known stage of pregnancy at different intervals after it has been given an intracardiac pulse-dose of tritiated thymidine, the phase of the mitotic-differentiating cell cycle in which the fetal cell was at the time of irradiation can be estimated. In 13- and 14-day fetuses, the primitive cells are largely involved in proliferation. In contrast, in later stage fetuses (such as 17 through 19 days) the majority of the vulnerable primitive cells are in $\mathrm{G} 1$ phase and migrating toward their destinations.

The major implications of the experiments, altogether, are that the mammalian fetus can recover to a remarkable degree from destructive injury by regulative regeneration, and it is a reasonable assumption that this capacity for recovery from injury may be a general response of mammals at some fetal stages.

\section{REFERENCES}

1. D'Amato, C. J., AND S. P. Hicks. 1980. Development of the motor system: effects of radiation on developing corticospinal neurons and locomotor function. Exp. Neurol. 70: $1-23$.

2. D'Amato, C. J., AND S. P. Hicks. 1980. Restitution of the central nervous system after destructive injury by prenatal irradiation in rats. Soc. Neurosci. Abstr. 6: 824 . 
3. D'Amato, C. J., ANDS. P. HiCKS. 1981. Hydrocephalus produced by prenatal irradiation. Soc. Neurosci. Abstr. 7: 467.

4. HICKs, S. P. 1954. Mechanisms of radiation anencephaly, anophthalmia, and pituitary anomalies. Repair in the mammalian embryo. Arch. Pathol. 57: 1-16.

5. HICKS, S. P., B. L. BROWN, AND C. J. D'AMATo. 1957. Regeneration and malformation in the nervous system, eye and mesenchyme of the mammalian embryo after radiation injury. Am. J. Pathol. 33: 459-481.

6. Hicks, S. P., AND C. J. D'AMATO. 1963. Malformation and regeneration of the mammalian retina following experimental radiation. Pages 45-51 in L. MICHAUX AND M. Feld Eds, Les phakomatoses cérébrales, Deuxième Colloque International, Malformations Congénitales de l'Encéphale. SPEI, Paris.

7. HICKS, S. P., AND C. J. D'AMATO. 1966. Effects of ionizing radiations on mammalian development. Pages 195-250 in D. H. M. WoolaM, Ed., Advances in Teratology, Vol. 1. Logos Press, London.

8. Hicks, S. P., AND C. J. D'Amato. 1968. Cell migrations to the isocortex in the rat. Anat. Rec. 160: 619-634.

9. Hicks, S. P., AND C. J. D'Amato. 1971. Visual function of rat retinas malformed by irradiation at birth. Society for Neuroscience First Annual Meeting. Program and Abstracts 13.17: 113 .

10. Hicks, S. P., AND C. J. D'Amato. 1977. Locating corticospinal neurons by retrograde axonal transport of horseradish peroxidase. Exp. Neurol. 56: 410-420.

11. HiCKS, S. P., AND C. J. D'AMATO. 1978. Effects of ionizing radiation on developing brain and behavior. Pages 35-72 in G. GotTlieB, Ed., Studies on the Development of Behavior and the Nervous System: Early Influences. Academic Press, New York.

12. Hicks, S. P., AND C. J. D'AMATO. 1980. Development of the motor system: hopping rats produced by prenatal irradiation. Exp. Neurol. 70: 24-39.

13. HiCKS, S. P., C. J. D'AMATO, AND D. L. JofTES. 1962. The nature of the radio-sensitive cells in the developing nervous system studied with tritiated thymidine. Pages 199-205 in B. GROSS AND V. ZELENY, Eds., Effects of Ionizing Radiation on the Nervous System. Int. Atomic Energy Agency, Vienna.

14. Hicks, S. P., C. J. D'Amato, And M. J. Lowe. 1959. The development of the mammalian nervous system. I. Malformations of the brain, especially the cerebral cortex, induced in rats by radiation. II. Some mechanisms of the malformations of the cortex. J. Comp. Neurol. 113: 435-469.

15. JACOBSON, M. 1978. Pages 10-13 in Developmental Neurobiology, 2nd ed. Plenum, New York. 